\title{
Repercusiones psicológicas de la disfunción eréctil sobre la autoestima y autoconfianza
}

\author{
A. Martín-Morales ${ }^{1}$, F. Meijide Rico ${ }^{2}$, J. I. García González ${ }^{3}$, L. Regadera Anechina ${ }^{4}$, \\ M. Manero Font ${ }^{5}$ \\ ${ }^{1}$ Hospital Carlos Haya. Málaga, ${ }^{2}$ Clínica Povisa. Vigo, ${ }^{3}$ Hospital de Móstoles. Madrid. \\ ${ }^{4}$ GlaxoSmithKline. Madrid, ${ }^{5}$ Q.F. Bayer.
}

Actas Urol Esp 2005; 29 (5): 493-498

\section{RESUMEN \\ REPERCUSIONES PSICOLÓGICAS DE LA DISFUNCIÓN ERÉCTIL SOBRE LA AUTOESTIMA Y AUTOCONFIANZA}

Introducción: La disfunción eréctil (DE) está causada por una amplia gama de factores orgánicos, psicológicos, psiquiátricos, interpersonales y farmacológicos. En numerosas publicaciones se menciona la pérdida de autoestima como un efecto colateral de la DE siendo muy probable también una afectación de la autoconfianza del sujeto. El objetivo del siguiente estudio fue evaluar la autoestima y autoconfianza de sujetos con DE y compararla con un grupo de sujetos sin DE.

Material y Métodos: Estudio epidemiológico, transversal, observacional, comparativo y multicéntrico. Se seleccionaron 405 sujetos varones mayores de 18 años en los que el médico de Atención Primaria sospechara que pudieran padecer DE. Todos los participantes debían cumplimentar de forma autoaplicada la versión española del cuestionario de autoestima de Rosenberg y la adaptación cultural al español del Cuestionario de autoconfianza de Johnson y McCoy. Después fueron remitidos a un urólogo que confirmaba el diagnóstico de DE ("gold Standard").

Resultados: El análisis estadístico mostró que los sujetos afectados de DE obtenían puntuaciones significativamente inferiores en las escalas de autoconfianza y autoestima que los sujetos $\sin \mathrm{DE}(\mathrm{p}<0.01)$.

Discusión: Los resultados ponen de manifiesto la pérdida de autoconfianza y autoestima que sufren los pacientes afectados de DE.

Palabras Clave: Disfunción Eréctil. Autoestima. Autoconfianza. Rosenberg. Cuestionarios.

\section{ABSTRACT}

PSYCHOLOGICAL IMPACT OF ERECTILE DYSFUNCTION ON SELF-ESTEEM AND SELF-CONFIDENCE

Background and objective: Erectile dysfunction (ED) is caused by a large range of organic, psychological, psychiatric, interpersonal and pharmacological factors. Numerous scientific publications mention the loss of self-esteem as a collateral effect of ED, with a very probable affectation of the subject's self-confidence. The objective of this study was to evaluate the self-esteem and self-confidence of subjects with ED and to compare them with a group of non-ED subjects.

Subjects and Method: An epidemiological, cross-sectional, observational, comparative and multicentric study was conducted. General Practitioners selected 405 men older than 18 years in which they suspected ED. All the participants had to complete the self administered form of the Spanish version of the Rosenberg self-esteem scale and the Spanish culturally adapted version of the Johnson and McCoy self-confidence scale. After that, patients were referred to the urologist in order to confirm the ED diagnosis.

Results: The statistical analysis of the data showed that the subjects with ED obtained significantly lower scores in the self-confidence and self-esteem scales than the non-ED subjects $(\mathrm{p}<0.01)$.

Discussion: The results of this study show the loss of self-confidence and self-esteem that suffer patients with ED.

Keywods: Erectile Dysfunction. Self-Esteem. Self-Confidence. Rosenberg. Questionnaires. 
$\mathrm{L}^{\mathrm{a}}$ a disfunción eréctil (DE) se define como la incapacidad persistente o recurrente para conseguir o mantener la suficiente rigidez del pene que permita una relación sexual satisfactoria $^{1}$. La DE está causada por una amplia gama de factores orgánicos, psicológicos, psiquiátricos, interpersonales y farmacológicos. Los factores psicológicos pueden dar lugar a ansiedad en relación con el acto sexual, pérdida de autoestima y aumento de la ansiedad que a su vez empeora la depresión y/o el estrés, estableciendo así un círculo vicioso.

La DE es un problema de salud de alta prevalencia $^{2}$ entre los hombres.

Diversos estudios han mostrado que repercute de forma importante en la disminución de la calidad de vida del varón afectado y su pareja, en áreas como la estabilidad emocional o la felicidad y las relaciones sociales $^{3-4}$. La experiencia aportada por los pacientes indica también que la DE es un problema de salud que provoca un gran impacto emocional, que afecta a la vivencia de la masculinidad. Existen publicaciones donde se menciona la pérdida de autoestima como un efecto colateral de la $\mathrm{DE}^{5-7}$.

Independientemente de la calidad de vida y la autoestima, cada día es más evidente que la DE se puede comportar como síntoma centinela de otras enfermedades (diabetes, hipertensión arterial, etc), y que está estrechamente relacionada con la evolución de enfermedades cardiovasculares, pudiendo actuar como marcador de la progresión de la enfermedad ${ }^{8}$. En muchos casos, los mecanismos fisiopatológicos de la DE son comunes a los implicados en la enfermedad vascular. Los hombres con enfermedad coronaria probada tienen una alta incidencia de DE y en algunos, el inicio de la DE anticipa los síntomas y diagnóstico de enfermedad coronaria ${ }^{9}$.

Los instrumentos utilizados para evaluar el impacto psicológico de la DE en los sujetos que la padecen incluyen, fundamentalmente, cuestionarios genéricos de calidad de vida relacionada con la salud, escalas de depresión y cuestionarios de autoestima. Sin embargo, hasta el momento no se ha publicado ningún estudio comparativo de sujetos con DE y sujetos sin DE en el que se evalúen específicamente estos dos conceptos con escalas ad-hoc.
El objetivo de este estudio fue evaluar la autoestima y autoconfianza de sujetos con DE y compararla con un grupo de sujetos sin DE. Es de interés realizar la medición de este tipo de variables teniendo en cuenta la aproximación biopsicosocial que requiere la $\mathrm{DE}$.

\section{MATERIAL Y MÉTODOS}

Se diseñó un estudio epidemiológico, transversal, observacional, comparativo y multicéntrico, denominado Proyecto ESTIMA (Estudio Transversal para detectar la disfunción eréctil y evaluar su Impacto sobre la Autoconfianza y autoestima). Todos los sujetos debían cumplimentar los cuestionarios del estudio, en la consulta del médico de atención primaria (AP). Para poder clasificar a los participantes en sujetos con DE o sujetos sin DE, el médico de AP debía remitir a todos los sujetos incluidos a una visita con el urólogo. Éste realizaba una historia clínica completa para confirmar o descartar el diagnóstico de DE.

El estudio contó con la participación de 13 médicos especialistas en urología y andrología y 92 médicos de AP en España. El protocolo obtuvo la aprobación del Comité Ético de Investigación Clínica (CEIC) del Hospital Carlos Haya de Málaga.

Se seleccionaron sujetos en las consultas de los médicos de AP, durante el periodo comprendido entre diciembre de 2002 y junio de 2003. Fueron candidatos a ser incluidos en el estudio todos aquellos varones mayores de 18 años en los que el médico sospechara que pudieran padecer la enfermedad, basándose en los síntomas que presentara el sujeto o que estuvieran recogidos en su historia clínica, o bien, en la información que solicitara el sujeto al médico. Debían mantener una relación heterosexual estable de más de seis meses de duración. Fueron excluidos los sujetos previamente diagnosticados de DE o que estuviesen recibiendo tratamiento para la DE y los que el médico de AP considerase necesario que iniciasen tratamiento antes de la visita al urólogo.

Antes de iniciar cualquier procedimiento específico del estudio se solicitó el consentimiento informado por escrito a los participantes.

Los participantes debían cumplimentar de forma autoaplicada la versión española del cues- 
tionario de autoestima de Rosenberg ${ }^{10-11}$ y la adaptación cultural al español del Cuestionario de autoconfianza de Johnson y McCoy ${ }^{12},{ }^{13}$

El cuestionario de autoconfianza consta de 18 items medidos mediante una escala de Likert de 7 puntos donde las respuestas oscilan desde el "Total acuerdo" 7 al "Total desacuerdo" 1 . La autoconfianza se entiende como el grado de confianza que tiene un individuo al realizar una determinada tarea o actividad. Las preguntas hacen referencia a lo que la persona piensa sobre sí misma y en la situación actual.

El cálculo de la puntuación global se obtiene mediante la suma de todos sus ítems de forma que una puntuación de 126 significa máxima autoconfianza y una puntuación de 18 una mínima autoconfianza.

Se utilizó la versión validada y traducida al español del cuestionario de autoestima de Rosenberg. Este cuestionario mide la autoestima global y está formado por 10 preguntas con cuatro alternativas de respuesta mediante una escala de Likert que va desde el "Totalmente de acuerdo" 4 al
"Totalmente en desacuerdo" ${ }^{\text {. Aquellos sujetos }}$ con puntuación global de 40 contaban con la máxima autoestima.

El análisis estadístico incluyó un análisis descriptivo de las características de los sujetos con DE y los sujetos sin DE mediante tablas de frecuencia para las variables de tipo nominal y medidas de tendencia central y dispersión para las variables continuas. Se efectuaron análisis de normalidad de la puntuación global de las escalas de autoestima y autoconfianza mediante el test de Shapiro-Wilks. Para la comparación entre ambos grupos se utilizó la prueba de la t de Student bajo los supuestos de normalidad, o en su defecto, su equivalente no paramétrico, test de la U de Mann-Whitney. Para la comparación de tablas de contingencia, entre ambos grupos, el análisis consistió en la utilización del test de la Chi-cuadrado, o en su defecto, el test de Fisher.

\section{RESULTADOS}

Descripción de la muestra. Fueron reclutados 405 pacientes (Fig. 1). De entre los sujetos que

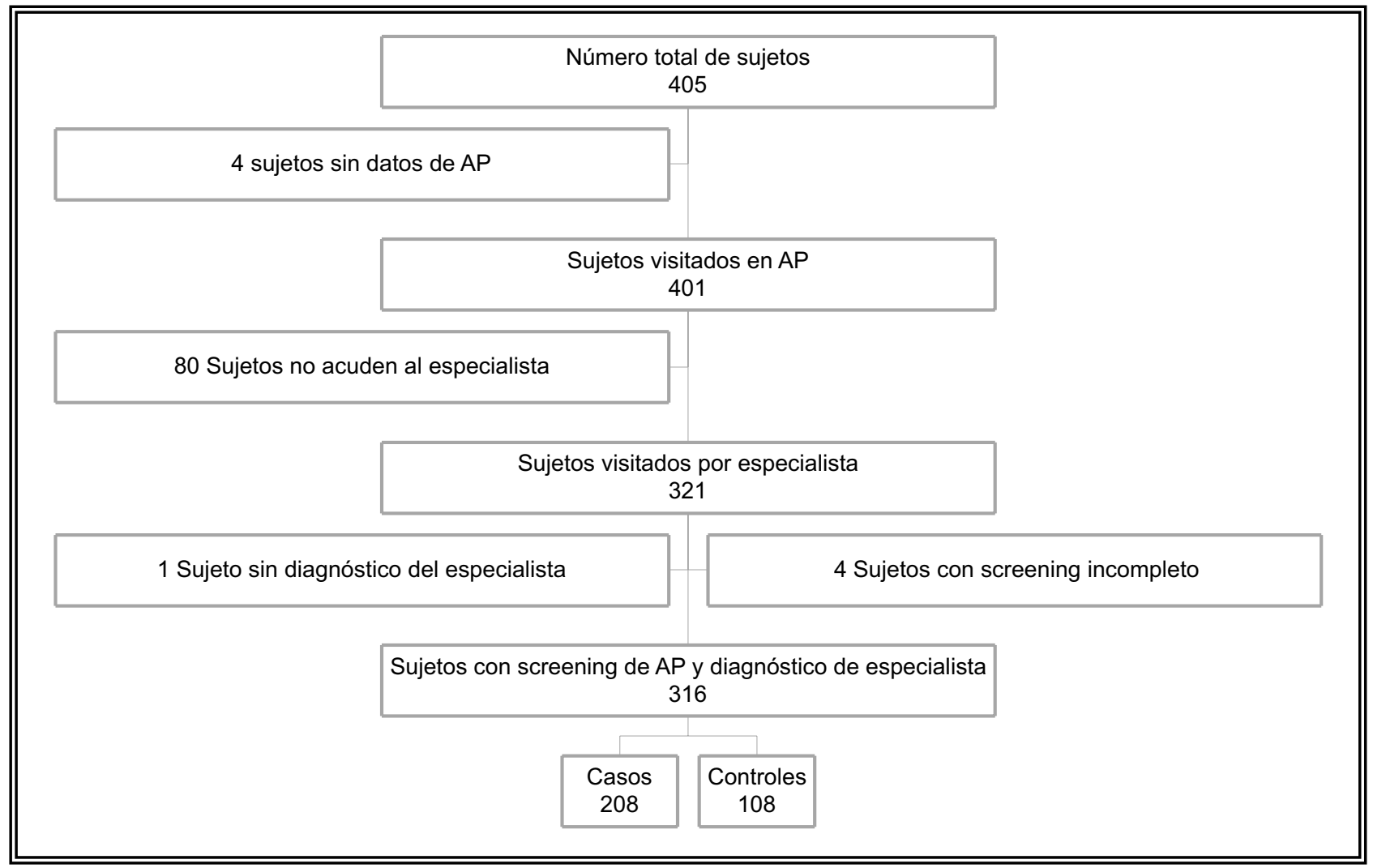

FIGURA 1. Distribución de la muestra de sujetos. 
respondieron a la pregunta de edad y acudieron a la visita del especialista para que confirmara su diagnóstico, 108, resultaron ser sujetos que no padecían la enfermedad. Éstos tenían edades (media \pm DE) comprendidas entre 19 y $80(52,9 \pm$ $11,5)$ años. El resto, 208 sujetos, de edades entre 20 y $76(57,3 \pm 10,0)$ años fueron sujetos en los que el urólogo confirmó el diagnosticó de DE.

Se observó una mayor proporción de diabéticos entre los sujetos con DE (23\% vs $11 \%$, $\mathrm{p}=0,014$, test de la Chi-cuadrado), no encontrándose diferencias en el resto de factores de riesgo examinados (hipertensión arterial, hipercolesterolemia, depresión, patología prostática y medicación crónica). Ambos grupos son por tanto comparables y caracterizándose fundamentalmente por la presencia o no de la DE. La Figura 2 muestra la distribución por grupos de los factores de riesgo.

Escala de autoconfianza. Se dispuso de un total de 313 individuos que contaban con el cuestionario de autoconfianza correctamente cumplimentado y el diagnóstico del especialista. La puntuación (media $\pm \mathrm{DE}$ ) de autoconfianza en los sujetos evaluados fue de $86,4 \pm 15,8$. En la Tabla 1 se muestra una descriptiva completa de la puntuación del cuestionario de autoconfianza según el diagnóstico del especialista.

Las puntuaciones de autoconfianza siguieron una distribución normal. Este análisis mostró diferencias estadísticamente significativas entre ambos grupos $(p=0,002)$. Los sujetos con DE obtuvieron una puntuación media de autoconfianza de 84.6 mientras que la puntuación media en los sujetos sin DE fue de 90,0.

Escala de autoestima. 315 individuos completaron el cuestionario de autoestima de Rosenberg y obtuvieron el diagnóstico del especialista. La puntuación de autoestima (media $\pm \mathrm{DE}$ ) en los sujetos evaluados fue de 31,4 $\pm 4,9$. En la Tabla 1 se muestra una descriptiva de la puntuación del cuestionario de autoestima en los dos grupos de sujetos.

Las puntuaciones de autoestima no siguieron una distribución normal. Este análisis mostró diferencias estadísticamente significativas $(\mathrm{p}<$ $0,001)$, siendo mayor la autoestima de los sujetos sin DE que los sujetos con DE (33,2 vs 30,5, respectivamente).

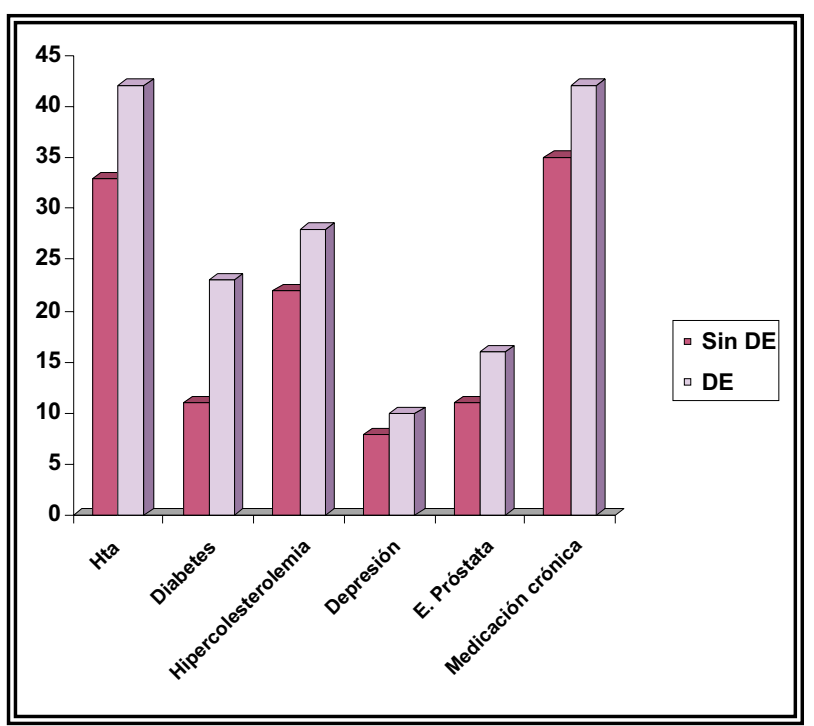

FIGURA 2. Distribución de los factores de riesgo por grupos.

El análisis de correlación de Pearson mostró una asociación positiva entre las puntuaciones de la escala de autoconfianza y la de autoestima $(\mathrm{r}=0,60, \mathrm{p}<0,001)$.

\section{DISCUSIÓN}

El principal objetivo de este estudio ha sido describir y analizar la repercusión de la DE sobre la autoestima y autoconfianza de los pacientes afectados respecto a sujetos sin DE. La autoestima se encuentra asociada a ansiedad y depresión ${ }^{14-15}$. Individuos con baja autoestima tienden a percibir de forma exagerada aquellas consecuencias negativas que se derivan de sus fracasos conductuales pudiendo llegar a un estado depresivo ${ }^{16}$.

En concordancia con otros estudios ${ }^{17,18}$ se confirma que la DE se asocia a unos niveles de autoestima disminuidos. Además, esta relación se observa también consistentemente sobre la autoconfianza.

Aunque el diseño del estudio no permite establecer una relación causa-efecto entre la DE y la autoestima y la autoconfianza, sí permite concluir que los pacientes que padecen DE presentan valores inferiores de estas dimensiones psicológicas que los pacientes, de similares características, que no padecen esta condición.

Por todo ello, es posible plantear que la DE no afecta exclusivamente a la función sexual del paciente sino que se transfiere a otras áreas de 
Tabla 1

Descriptiva de las puntuaciones de autoconfianza y autoestima

\begin{tabular}{|c|c|c|c|c|c|}
\hline & & $\mathbf{N}$ & Media \pm DE & IC $95 \%$ & $\begin{array}{l}\text { Mediana } \\
\text { (Mín-Máx) }\end{array}$ \\
\hline \multirow[t]{2}{*}{ Autoconfianza } & $\begin{array}{l}\text { Disfunción } \\
\text { Eréctil }\end{array}$ & 205 & $84.6 \pm 16.5$ & $82.3-86.8$ & $\begin{array}{c}84 \\
(41-122)\end{array}$ \\
\hline & $\begin{array}{l}\text { Sin Disfunción } \\
\text { Eréctil }\end{array}$ & 108 & $90.0 \pm 13.7$ & $87.4-92.6$ & $\begin{array}{c}91 \\
(51-120)\end{array}$ \\
\hline \multirow[t]{2}{*}{ Autoestima } & $\begin{array}{l}\text { Disfunción } \\
\text { Eréctil }\end{array}$ & 207 & $30.5 \pm 4.8$ & $29.8-31.2$ & $\begin{array}{c}30 \\
(15-40)\end{array}$ \\
\hline & $\begin{array}{l}\text { Sin Disfunción } \\
\text { Eréctil }\end{array}$ & 108 & $33.2 \pm 4.6$ & $32.3-34.0$ & $\begin{array}{c}33 \\
(19-40)\end{array}$ \\
\hline
\end{tabular}

DE: Desviación estándar; IC: Intervalo de Confianza; Mín: Valor Mínimo; Máx: Valor Máximo

su vida. La DE puede hacer abandonar al paciente la práctica sexual y las relaciones sociales, causando un perjuicio en su estado de salud global ${ }^{19}$.

\section{Agradecimientos}

A los investigadores del proyecto ESTIMA.

Urólogos: Juan José Lobato Encinas (H. General Alicante. Alicante), Eduardo Ruiz Castañé (Fundación Puigvert. Barcelona), Joan Prats López (H. Parc Taulí de Sabadell. Barcelona), Adrián de la Fuente (H. Clínico de Granada. Granada), Juan Pablo Sanz Jaca (H. Comarcal de Bidasoa. Hondarribia), Jesús Isidoro García González (H. de Móstoles. Madrid), Luis Fiter Gómez (H. Severo Ochoa de Leganés. Madrid), Antonio Martín Morales (H. Carlos Haya. Málaga), Javier Regadera Seijas (H. Central de Asturias. Oviedo), Esteban Blanco Palenciano (H. V. De la Macarena. Sevilla), Francisco Ramada Benlloch (H. General de Valencia. Valencia), José Ramón Cortiñas González (H. Clínico Universitario de Valladolid. Valladolid), Fernando Meijide Rico (Clínica Povisa. Vigo).

Médicos de Atención Primaria: Remedios Alarcón Barbero (C.S Florida. Alicante), José Rafael Berná Salas (C.S Los Angeles. Alicante), Carlos Ojea Arrendó y Francisco Llorca Catarla (C.S San Vicente. Alicante), Julio González Algas y Miguel Angel Fuentes Pérez (C.A.P Maragall. Barcelona), Carmen Berbel Bolivar (C.S Almuñecar. Granada), Manuel Alonso Ródenas (C.S Casería de Montijo. Granada), Carlos Sanchez Tovar (C.S Deifontes. Granada), José Antonio López Sánchez (C.S Loja. Granada), Antonio Pineda García (C.S Macarena. Granada), Juan Tomas Gilabert (C.S Mirasierra. Granada), José Luis Martín Manzano (C.S Salvador Caballero. Granada), Carmen Gonzalez García (C.S Tiena. Granada), José María Etxebeste Inzagaray,Fco Javier García Chivite, Antonio García Rodríguez, Agurne Linacisoro Ibarra, Enrique Pérez-Mosso Neninger, Luis Unzueta Salvador y
Arantxa Calvo Elizazu (C.S Dumboa. Irán), Jose Pérez Sanchez (C.S Loranca. Leganés), Javier Rodriguez Peso (C.S Pedroches. Leganés), Jesús Luengo Agüero (Clínica Belén. Leganés), Melitón Blasco Oliete (EAP Humanes. Leganés), Julio Sanchez Salvador (EAP M ${ }^{\mathrm{a}}$ Angeles López. Leganés), Tomas Rodelgo Cambronero (EAP Pedroches. Leganés), Fco Javier Huerta Dante (M. Jesus Hereza. Leganés), Luis Miguel de la Morena González (C.S Arroyo de la Miel. Málaga), Alvaro Milán Fernándezy Francisco Tabeada González (C.S Ciudad Jardín-29014. Málaga), Vicente Izco López (C.S Limonar. Málaga), Pedro García Fernández y Diego Lara Navarro (C.S Nueva Málaga. Málaga), Ignacio Martínez Mérida (C.S Puerta Blanca. Málaga), Enrique Martos Gutiérrez (C.S San Miguel. Málaga), Inmaculada Sanchez Hernández (C.S Valle de Abdalagis. Málaga), Josefina Aparicio Velasco, Jose Manuel Méntrida Rodríguez y M M $^{\mathbf{a}}$ Carmen Marin Osorio (C.S Alcalde Bartolomé. Móstoles), Juan Carlos Muñoz García (C.S Azorin. Móstoles), Francisco Rodrigo Jiménez y Alfredo Falcó Sales (C.S Barcelona. Móstoles), Daniel Serrano Tomas, Gregorio Perdices Pinilla y Pablo Romero Martínez (C.S Dos de Mayo. Móstoles), Gonzalo Zarate Valenzuela (C.S Presentación Sabio. Móstoles), Ramón Alonso Gómez (C.S Noreña. Oviedo), Ignacio González Escandón (C.S Posada de Llanera. Oviedo), Jose Antonio Fernández Fernández (C.S Pumarin. Oviedo), Eduardo Murias Siñeriz (C.S Sta. Eulalia. Oviedo), Joaquín Cuetos Alvarez (Consultorio de Valdesoto. Oviedo), Miguel Angel Fernández Fernández (Jefatura Superior. Oviedo), Javier Ignacio Garrido Peon (Sanidad Guardia Civil. Oviedo), Maria Jesús Cubells Larrosa (ABS Lepant. Sabadell), Francesc Solé Llorens (C.S Privada. Sabadell), Antonio Moya Moya y Antonio Salido Cano (CAP Castellar del Vallès. Sabadell), Miguel González Herrero y Esperanza Fernández Barrio (CAP Concordia. Sabadell), Joseph Palou Torrentó (CAP Rambla 
Sabadell. Sabadell), Rufino Orús Loncá (ICS Mutuam. Sabadell), Jose Manuel Toro Barragán y Enrique Diaz Monge (C.S Coria del Río. Sevilla), Manuel Cancio Luque (C.S Dr. Fleming. Sevilla), Adán González Huambos (C.S El Porvenir. Sevilla), Blas Poyatos Poyatos (C.S Polígono Norte. Sevilla), Juan Manuel Miras Ruiz y Jose Joaquín Masa Sanchez (C.S San José Rinconada. Sevilla), Antonio Lemus Gallego (C.S San Pablo. Sevilla), Carlos Almendro Padilla, Joaquin Casado Gonzalez, José Isidoro Martínez Olivares, Miguel Angel Furió Soriano, Fernando Roselló Roca, $\mathrm{M}^{\mathrm{a}}$ Dolores Alfonso Doménech y Concha Estellés Dasí (C.S Torrent. Valencia), Maㅜ Teresa García Gonzalo y Amaya Narbaiza Garzabal (C.S Circular. Valladolid), Ricardo García Alonso (C.S de Olmedo. Valladolid), Ma Concepción Gil San Martín (C.S Delicias I. Valladolid), Pedro García de Padilla (C.S Pilanza. Valladolid), Jose Carlos Minguez Villaiz (C.S Tortola. Valladolid), Manuel González-Alegre Díez, Gerardo Miniño Couto y Enrique Cobas Varela (C.S Beiramar. Vigo), Federico Cardelle García (C.S Casa de Mar. Vigo), Lino Fontaína Pérez (C.S Coia. Vigo), Carmen Pache Muínos y Rosa Ledo Rivas (C.S Coruxo. Vigo), Manuel Bayón Meleiro (C.S López Mora. Vigo), Sagrario Franco Malvar (C.S. Val de Miñor. Vigo).

\section{REFERENCIAS}

1. Documento de Consenso sobre Disfunción Eréctil. Elaborado por 12 entidades científicas. Marzo 2002.

2. Martín-Morales A, Sánchez-Cruz JJ, Sáenz de Tejada I, Rodríguez-Vela L, Jiménez-Cruz JF, Burgos-Rodríguez R. Prevalence and independent risk factors for erectile dysfunction in Spain: results of the Epidemiologia de la Disfuncion Erectil. J Urol 2001; 166(2):569-575.

3. Althof SE, Cappelleri JC, Shpilsky A, Stecher V, Diuguid C, Sweeney M, et al. Treatment responsiveness of the selfesteem and relationship questionnaire in erectile dysfunction. Urology 2003; 61(5):888-892.

4. Latini DM, Penson DF, Colwell HH, Lubeck DP, Mehta SS, Henning JM, et al. Psychological impact of erectile dysfunction: validation of a new health related quality of life measure for patients with erectile dysfunction. J Urol 2002; 168(5):2086-2091.

5. Intili H, Nier, D. Self-esteem and depression in men who present with erectile dysfunction. Urol Nurs 1998; 18(3):185-187,208.

6. Willke RJ, Yen W, Parkerson GR, Linet OI, Erder MH, Glick HA. Quality of life effects of alprostadil therapy for erectile dysfunction: results of a trial in Europe and South Africa. Int J Impot Res 1998; 10(4):239-246.
7. Smith AD. Psychological factors in the multidisciplinary evaluation and treatment of erectile dysfunction. Urol Clin North Am 1988; 15(1):41-51.

8. Kirby M, Jackson G, Betteridge J, Friedli K. Is erectile dysfunction a marker for cardiovascular disease? Int $\mathrm{J}$ Clin Pract 2001; 55(9):614-618.

9. O'Kane PD, Jackson G. Erectile dysfunction: is there silent obstructive coronary artery disease? Int J Clin Pract 2001; 55(3):219-220.

10. Sotelo MJ. Sex differences in self-concept in Spanish secondary school students. Psychol Rep 2000; 87:731-734.

11. Rosenberg M. Society and the adolescent self-image. Princeton, NJ: Princeton Univer. Press 1965.

12. Johnson W, McCoy N. Self-confidence, self-esteem, and assumption of sex role in young men and women. Percept Mot Skills 2000; 90:751-756.

13. Martín-Morales A, Manero M, Regadera L, Artés M. A new instrument to measure the psychological impact of erectile dysfunction. Validation of a Spanish Version of the Johnson and McCoy's Self-Confidence Scale [abstract]. ISPOR $6^{\text {th }}$ Annual European Congress; 2003, November 911; Barcelona.

14. Parry G, Brewin CR. Cognitive Style and Depression: symptom-related, event-related or independent provoking factor? Br J Clin Psychol 1988; 27:23-35.

15. Greenberg J, Solomon S, Pyszczynski T, Rpsembñatt A, Burling J, Lyon D, et al. Why Do people need self-esteem? Converging evidence that self-esteem serves and anxietybuffering function. J Pers Soc Psychol 1992; 63(6):913922.

16. Roberts JE, Monroe SM. Vulnerable self-esteem and depressive symptoms: prospective findings comparing three alternative conceptualizations. J Pers Soc Psychol 1992; 62(5):804-812.

17. Althof SE, Cappelleri JC, Shpilsky A, Stecher V, Diuguid C, Sweeney $M$ et al. Treatment responsiveness of the SelfEsteem And Relationship questionnaire in erectile dysfunction. Urology 2003; 61(5):888-892.

18. Latini DM, Penson DF, Colwell HH, Lubeck DP, Mehta SS, Henning JM et al. Psychological impact of erectile dysfunction: validation of a new health related quality of life measure for patients with erectile dysfunction. J Urol 2002; 168(5):2086-2091.

19. NIH Consensus Development Panel of Impotence. Impotence. JAMA 1993: 270:83-90.

Dra. A. Muñoz

Adelphi Targis.

C/ Rosellón, 25, Bajos $1^{\text {a }}$

08029 Barcelona

(Trabajo recibido el 6 abril de 2005) 